\title{
Low skeletal muscle mass is associated with arterial stiffness in community- dwelling Chinese aged 45 years and older
}

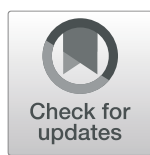

\author{
Mingzhe Yang ${ }^{1 \dagger}$, Xuguang Zhang $^{1 \dagger}$, Zhenkai Ding ${ }^{1}$, Feijie Wang ${ }^{1}$, Yufang Wang ${ }^{1}$, Changya Jiao ${ }^{1}$ and \\ Jie-Hua Chen ${ }^{1,2^{*}}$ (D)
}

\begin{abstract}
Background: Evidence suggests that body composition has impact on arterial stiffness. However, evidence in Chinese are limited, and results remain controversial. The aim of our study is to investigate whether skeletal muscle mass is associated with arterial stiffness in Chinese community-dwelling men and women aged 45 years and older.

Methods: In this cross-sectional study, 20,477 participants (age range: 45-80 years, 68.8\% women) were included in the analysis. Brachial-ankle pulse wave velocity (baPWV), an indicator of arterial stiffness was measured using a waveform device. Total muscle mass and muscle mass of arm, leg and trunk were measured by bioelectrical impedance analysis. Height and weight were measured and appendicular skeletal muscle mass index (ASMI) was calculated as appendicular skeletal muscle mass (sum of arm and leg muscle mass) divided by height square.

Results: After adjustment for age, body fat percentage, systolic blood pressure and diastolic blood pressure, ASMI was negatively associated with baPWV $[\beta$ (SE) for men: $-0.208(0.016), p<0.0001$; for women: -0.245 $(0.012), p<0.0001]$. High ASMI was a protective factor for the presence of arterial stiffness (defined as baPWV) [OR (95\%Cl) for men: 0.730 (0.682, 0.782), $p<0.0001$; women: 0.634 (0.593, 0.677), $p<0.0001]$. Similar associations were found between quantity of muscle mass (total and appendicular muscle mass, muscle mass of arm, leg and trunk) and arterial stiffness in men and women after further adjustment for height (all $p<0.0001$ ).
\end{abstract}

Conclusion: Low skeletal muscle mass is associated with increased risk of arterial stiffness in Chinese communitydwelling adults aged 45 years and older.

Keywords: Skeletal muscle mass, Body composition, Pulse wave velocity, Arterial stiffness

\section{Background}

Cardiovascular disease (CVD) remains to be the top cause of death in China and brings out huge medical and financial burden [1]. Arterial stiffness, a reflection of health status of both central and peripheral arteries, has strong predictive value for future cardiovascular events and all-cause mortality [2, 3]. Pulse wave velocity

\footnotetext{
* Correspondence: siyanpijiehua@gmail.com

${ }^{\dagger}$ Mingzhe Yang and Xuguang Zhang contributed equally to this work and shared first authorship.

${ }^{1}$ Nutrition and Health Research Centre, By-Health Co. Ltd, No. 3 Kehui 3rd Street, No. 99 Kexue Avenue Central, Science City, Huangpu District, Guangzhou 510633, China

${ }^{2}$ Department of Nutrition and Food Hygiene, School of Public Health, Southern Medical University, Guangzhou North Avenue 1838, Guangzhou 510515, People's Republic of China
}

(PWV), a standard and noninvasive indicator applied in many epidemiological and clinical studies for assessing arterial stiffness, has been confirmed as an independent predictor of future cardiovascular events [4-8]. Results from meta-analysis showed that every $1 \mathrm{SD}$ increase of the brachial-ankle pulse wave velocity (baPWV) was associated with a 1.19-fold increase in the risk of CVD [4].

Evidence suggests that body composition has impact on the prevalence and development of arterial stiffness [9-14]. More specifically, previous studies which explored the association between muscle mass and PWV showed consensus preliminarily. Significant negative associations were found between muscle mass indices and PWV in studies among apparently healthy elderly of Chinese [15], Japanese [16, 17] and American with 
multi-race [18]. Similar results were found among patients of type 2 diabetes [19] and peritoneal dialysis [20]. However, evidence in Chinese population are limited, and results remain controversial among different gender and for regional muscle mass (arm, leg and trunk).

Possible pathways for the association between low muscle mass and arterial stiffness might lie in insulin resistance and chronic inflammation. Loss of muscle mass was considered as a cause of insulin resistance by impairing glucose metabolism [21], and evidence showed that insulin resistance could determine the arterial elastic properties [22]. On the other hand, it had been suggested that some inflammatory cytokines were associated with an increased risk of sarcopenia [23], and some inflammatory markers had been examined as causal agents in the pathogenesis of arterial stiffness [24].

The aim of this study was to explore the associations between muscle mass indices (total and appendicular muscle mass, appendicular skeletal muscle mass index, and muscle mass of arm, leg and trunk) and arterial stiffness (defined as baPWV) in community-dwelling Chinese men and women aged 45 years and older.

\section{Methods}

\section{Study design and study population}

Data of the participants were from the Screening of Chronic Diseases in Chinese Adults (SCDCA) study, a cross-sectional study started in 2017, which aims to screen four chronic diseases (obesity, osteoporosis, arterial stiffness and age-related macular degeneration) and explore the influencing factors among Chinese community-dwelling adults in urban areas from 24 administrative regions. According to the population size and cooperation, we included several cities/areas in each administrative region. In each city/town, the communities for screening were enrolled using cluster random sampling. The research staff were divided into five groups, each group was equipped with mobile health check-up vehicle, a specially designed truck carried research instruments to designated sampling cities. Eligible participants in this study were men and women: 1) aged above 18 years old, 2) who have resided locally for more than 5 years, 3) who were cooperative and volunteered. The following participants were excluded: 1) pregnant and lactating women, 2) participants with major organ diseases, including heart, liver or kidney disease, 3) participants with mental diseases, 4) unable to carry out body examination due to disabilities. In the present analysis, participants from March 2017 to May 2018 were enrolled and the following were further excluded: 1) aged under 45 years, 1) participants with peripheral artery disease whose ankle-brachial index $(\mathrm{ABI}) \leq 0.9$ [25], 2) participants with incomplete information of body composition, arterial stiffness and covariates. Thus, a total of 20,477 participants were included in the present analysis. The study was approved by Ethics Committee of Chinese Clinical Trial Registry. All participants gave written informed consent.

\section{Anthropometric measurements}

Height and weight were measured by trained medical workers according to standard procedures, with subjects wearing light indoor clothing and barefoot. Height was measured to the nearest $0.1 \mathrm{~cm}$ and weight was measured to the nearest $0.1 \mathrm{~kg}$. BMI (body mass index) was calculated as weight $(\mathrm{kg})$ divided by height squared $\left(\mathrm{m}^{2}\right)$.

\section{Measurements of body composition indices}

The quantity of total skeletal muscle mass (SMM), muscle mass of the left arm (LAMM), right arm (RAMM), left leg (LLMM), right leg (RLMM) and trunk (TMM) and the percentage of body fat (BFP) were measured using a dual bioelectrical impedance analyzer (IOI 353; Jawon, Korea). Bioelectrical impedance analysis (BIA) is widely used in the detection of body composition and this instrument were used in several studies $[26,27]$. Appendicular skeletal muscle mass (ASM, kg) was defined as the sum of the arm and leg muscle mass on both sides. Appendicular skeletal muscle mass index (ASMI, $\mathrm{kg} / \mathrm{m}^{2}$ ) was calculated as ASM divided by height square.

\section{Measurements of arterial stiffness indices and blood pressure}

In this study, the baPWV, mean arterial pressure (MAP), pulse pressure (PP), upstroke time (UT) as well as the brachial and ankle blood pressures on the left and right sides of the subjects were measured with a plethysmography apparatus (BP-203RPE III; Omron, Japan) after rested in a supine position for 5 min. Evidence showed that the validity and reproducibility of this instrument is considerably high and suitable for screening vascular damages in a large population [28]. baPWV, MAP, PP and UT were calculated as the mean of the left and right baPWV, MAP and UT values, respectively. Systolic blood pressure (SBP) and diastolic blood pressure (DBP) were calculated as the mean of the left and right brachial systolic and diastolic pressure, respectively. High baPWV (arterial stiffness) was defined as baPWV $\geq 1800 \mathrm{~mm} / \mathrm{s}$ [29].

\section{Data collection of other characteristics}

A standard questionnaire was used to collect each participant's age, gender, education level, medical history, and habits of smoking, drinking and exercise. Participants 
filled the questionnaire on a computer/smartphone under the supervision of trained research staff.

\section{Statistical analysis}

Categorical variables were described as number and percentage. Continuous variables with normal distribution were expressed using mean with standard deviation (SD), and non-normally distributed continuous variables were presented as median with 25th and 75th percentile. The participants were stratified into three groups by tertiles of the ASMI (men: $7.71 \pm 0.37 \mathrm{~kg} / \mathrm{m}^{2}, 8.53 \pm 0.22 \mathrm{~kg}$ / $\mathrm{m}^{2}, 9.78 \pm 0.73 \mathrm{~kg} / \mathrm{m}^{2}$; women: $6.77 \pm 0.34 \mathrm{~kg} / \mathrm{m}^{2}, 7.51 \pm$ $\left.0.18 \mathrm{~kg} / \mathrm{m}^{2}, \quad 8.41 \pm 0.57 \mathrm{~kg} / \mathrm{m}^{2}\right)$. Differences between groups were tested using Student's t-test or one-way ANOVA analysis for normally distributed continuous variables and Kruskal-Wallis test for continuous variables with non-normal distribution. Pearson's correlation analysis was used to access the correlation between muscle mass indices (ASMI, ASM, SMM, LAMM, RAMM, LLMM, RLMM and TMM) and baPWV. The association of muscle mass indices with baPWV and the association of mass indices with arterial stiffness (normal / high, defined as baPWV) were carried out by multivariate linear regression analysis and multiple logistic regression analysis, respectively. The regression models testing ASMI with baPWV / arterial stiffness were performed after adjusting for age, body fat, SBP and DBP. The models testing quantity of muscle mass (SMM, ASM, LAMM, RAMM, LLMM, RLMM, TMM) with baPWV / arterial stiffness were further adjusted for body height. All analyses were conducted using SAS version 9.1 (SAS Institute, Inc., Cary, NC, USA). A $p$ value $<0.05$ was considered statistically significant.

\section{Results}

The mean age of participants included in the present analysis was $61.25 \pm 8.22$ years (age range: $45-80$ years), $68.79 \%$ were women. Compared with women, men were older and had higher BMI, SMM, AMM, LAMM, RAMM, LLMM, RLMM, TMM, baPWV, SBP, DBP and ABI, however, their BFP, PP, MAP and UT were lower than that of women. Compared with women, the prevalence of diabetes and hypertension, the percentage of high education level, current smoker, current alcohol user and regular exercise were higher in men (all $p<0.05)$ (Table 1).

Characteristics of body composition and artery stiffness in men and women are presented in Table 2. The participants were divided into three groups according to ASMI tertiles (men: $7.71 \pm 0.37 \mathrm{~kg} / \mathrm{m}^{2}, 8.53 \pm 0.22 \mathrm{~kg} / \mathrm{m}^{2}$, $9.78 \pm 0.73 \mathrm{~kg} / \mathrm{m}^{2}$; women: $6.77 \pm 0.34 \mathrm{~kg} / \mathrm{m}^{2}, 7.51 \pm 0.18$ $\left.\mathrm{kg} / \mathrm{m}^{2}, 8.41 \pm 0.57 \mathrm{~kg} / \mathrm{m}^{2}\right)$. Both among men and women, higher ASMI was observed in participants who were younger and those with lower baPWV and MAP (all $p<$
0.0001). In contrast, participants with higher ASMI had higher BMI, ASM, SMM, LAMM, RAMM, LLMM, RLMM, TMM, SBP, DBP, PP, ABI and UT (all $p<0.05$ ). The highest BFP was found in median ASMI group (T2) for men and in high ASMI group (T3) for women.

Pearson's correlations between muscle mass indices and baPWV are shown in Table 3. Significant negative correlations were observed between muscle mass indices (ASMI, ASM, SMM, LAMM, RAMM, LLMM, RLMM, and TMM) and baPWV in men and women (all $p<0.0001$ ).

The results of the multiple linear regression showed that ASMI was negatively associated with baPWV $[\beta$ (SE) for men: - 0.208 (0.016), $p<0.0001$; women: - 0.245 (0.012), $p<0.0001]$ adjusted for age, body fat percentage, systolic blood pressure and diastolic blood pressure. Similar associations were found between quantity of muscle mass (ASM, SMM, LAMM, RAMM, LLMM, RLMM, and TMM) and baPWV in men and women after further adjustment for height (all $p<0.0001$ ) (Table 4).

Multiple logistic regression analysis showed that higher ASMI was a protective factor for the presence of arterial stiffness adjusted for age, body fat percentage, systolic blood pressure and diastolic blood pressure [OR (95\%CI) for men: 0.730 (0.682, 0.782), $p<0.0001$; women: $0.634(0.593,0.677), p<0.0001]$. Similar associations were found between quantity of muscle mass (ASM, SMM, LAMM, RAMM, LLMM, RLMM, and TMM) and arterial stiffness in men and women after further adjustment for height (all $p<0.0001$ ) (Table 5).

\section{Discussion}

The present study found that ASMI and quantity of muscle mass (total and appendicular muscle mass, muscle mass of arm, leg and trunk) were negatively associated with baPWV. High levels of these muscle mass indices were protective factors for the presence of arterial stiffness in Chinese community-dwelling men and women aged 45 years and older.

Recently, epidemiological data showed that the middle-aged population in North China had a high prevalence of arterial stiffness (high baPWV) [30]. Compared with this study, the prevalence of arterial stiffness in the present analysis was higher in men and approximate in women (men: $33.43 \%$, women: $26.63 \%$, data not shown). Meanwhile, the quantity of total skeletal muscle mass of Chinese adults was at a low level, especially for the elderly [31], which was comparable with our results.

The negative associations between SMM / ASM / ASMI and baPWV were found in previous studies [15, 17, 20]. Zhang et al. [15] found that ASMI was associated with baPWV in 1002 Chinese community dwelling participants aged 65 years and above, and this relation remains significant only in men in analysis of gender subgroup. Similar association was observed between 
Table 1 Characteristics of the study population by gender

\begin{tabular}{|c|c|c|c|}
\hline & Men & Women & $p^{a}$ \\
\hline N & 6390 & 14,087 & \\
\hline Age (years) & $62.87 \pm 8.07$ & $60.30 \pm 8.00$ & $<0.0001$ \\
\hline High education level (\%) & 14.76 & 11.05 & $<0.0001$ \\
\hline Current smokers (\%) & 31.84 & 2.27 & $<0.0001$ \\
\hline Current alcohol user (\%) & 40.30 & 8.03 & $<0.0001$ \\
\hline Regular exercise (\%) & 20.09 & 18.41 & $<0.0001$ \\
\hline Type 2 diabetes (\%) & 11.07 & 9.86 & $<0.0001$ \\
\hline Hypertension (\%) & 30.76 & 26.65 & $<0.0001$ \\
\hline Height (cm) & $168.6 \pm 5.88$ & $158.24 \pm 5.35$ & $<0.0001$ \\
\hline Weight (kg) & $69.78 \pm 10.52$ & $61.13 \pm 9.35$ & $<0.0001$ \\
\hline BMI $\left(\mathrm{kg} / \mathrm{m}^{2}\right)$ & $24.51 \pm 3.23$ & $24.39 \pm 3.40$ & 0.014 \\
\hline SMM (kg) & $49.36 \pm 6.18$ & $38.13 \pm 4.32$ & $<0.0001$ \\
\hline LAMM (kg) & $3.30 \pm 0.48$ & $2.50 \pm 0.33$ & $<0.0001$ \\
\hline RAMM (kg) & $3.31 \pm 0.49$ & $2.49 \pm 0.35$ & $<0.0001$ \\
\hline LLMM (kg) & $9.05 \pm 1.28$ & $7.00 \pm 0.93$ & $<0.0001$ \\
\hline RLMM (kg) & $9.06 \pm 1.27$ & $6.99 \pm 0.90$ & $<0.0001$ \\
\hline TMM (kg) & $24.65 \pm 2.78$ & $19.16 \pm 1.96$ & $<0.0001$ \\
\hline ASM (kg) & $24.72 \pm 3.47$ & $18.98 \pm 2.42$ & $<0.0001$ \\
\hline $\operatorname{ASMI}\left(\mathrm{kg} / \mathrm{m}^{2}\right)$ & $8.68 \pm 0.98$ & $7.57 \pm 0.78$ & $<0.0001$ \\
\hline BFP (\%) & $23.50(19.70,26.90)$ & $31.80(28.50,34.70)$ & $<0.0001$ \\
\hline baPWV (cm/s) & $1650.00(1449.00,1894.00)$ & $1567.00(1372.00,1822.00)$ & $<0.0001$ \\
\hline SBP $(\mathrm{mmHg})$ & $137.41 \pm 19.00$ & $135.87 \pm 20.63$ & $<0.0001$ \\
\hline DBP (mmHg) & $81.32 \pm 10.95$ & $78.34 \pm 11.14$ & $<0.0001$ \\
\hline $\mathrm{PP}(\mathrm{mmHg})$ & $58.00 \pm 12.19$ & $59.58 \pm 13.13$ & $<0.0001$ \\
\hline$A B \mid$ & $1.21 \pm 0.012$ & $1.18 \pm 0.10$ & $<0.0001$ \\
\hline MAP (\%) & $39.85 \pm 3.93$ & $42.50 \pm 3.46$ & $<0.0001$ \\
\hline UT (s) & $144.00(133.00,160.00)$ & $156.00(142.00,174.00)$ & $<0.0001$ \\
\hline
\end{tabular}

Values are percentage, mean \pm SD or medians (25th and 75 th percentile)

Abbreviations: BMI Body mass index, SMM Total muscle mass, LAMM Left arm muscle mass, RAMM Right arm muscle mass, LLMM Left arm muscle mass, RLMM Right leg muscle mass, TMM Trunk muscle mass, BFP total body fat percentage, ASM Appendicular skeletal muscle, $A S M I$ Appendicular skeletal muscle index, baPWV Brachial-ankle pulse wave velocity, SBP Systolic blood pressure, DBP Diastolic blood pressure, $P P$ Pulse pressure, $A B I$ Ankle brachial index, MAP Mean artery pressure, UT Upstroke time

${ }^{\text {a } T e s t ~ f o r ~ d i f f e r e n c e ~ b e t w e e n ~ g e n d e r ~ g r o u p s ~ w a s ~ p e r f o r m e d ~ u s i n g ~ t h e ~ C h i-s q u a r e ~ t e s t ~ f o r ~ c a t e g o r i c a l ~ v a r i a b l e s, ~ K r u s k a l-W a l l i s ~ t e s t ~ f o r ~ n o n-n o r m a l l y ~ d i s t r i b u t e d ~}$ continuous variables and $T$ test for normally distributed continuous variables

total skeletal muscle mass and baPWV among Chinese peritoneal dialysis patients [20], which was mainly contributed by female patients. Moreover, studies among Japanese women suggested that baPWV was a risk factor for the low level of ASMI [17]. In accordance with these studies, our results suggested that Chinese adults with low levels of SMM / ASM / ASMI had higher baPWV. Consistent results were observed both in men and women. Our findings from a large sample of Chinese community-dwelling population confirmed the hypothesis that low level of muscle mass is a potential risk factor of arterial stiffness. To our knowledge, this relation was proved in both genders for the first time. Possible reasons for the conflicting results between men and women included that: 1) the level of baPWV, muscle mass or other covariates were significantly different between gender groups, 2) the sample size in the subgroup was limited for examining the potential associations, 3) some androgens $[32,33]$ and the different distribution of body composition in men and women [34, 35] might have potential effect on this relation.

Several studies further explored the impact of regional muscle mass quantity on pulse wave velocity which showed controversial results. A study among multi-race American elderly reported that high cfPWV (carotidfemoral pulse wave velocity) was associated with lower leg and arm mass in all men and lower leg mass in white women [18]. However, among Asian Indians with 
Table 2 Characteristics of the study population according to ASMI tertiles

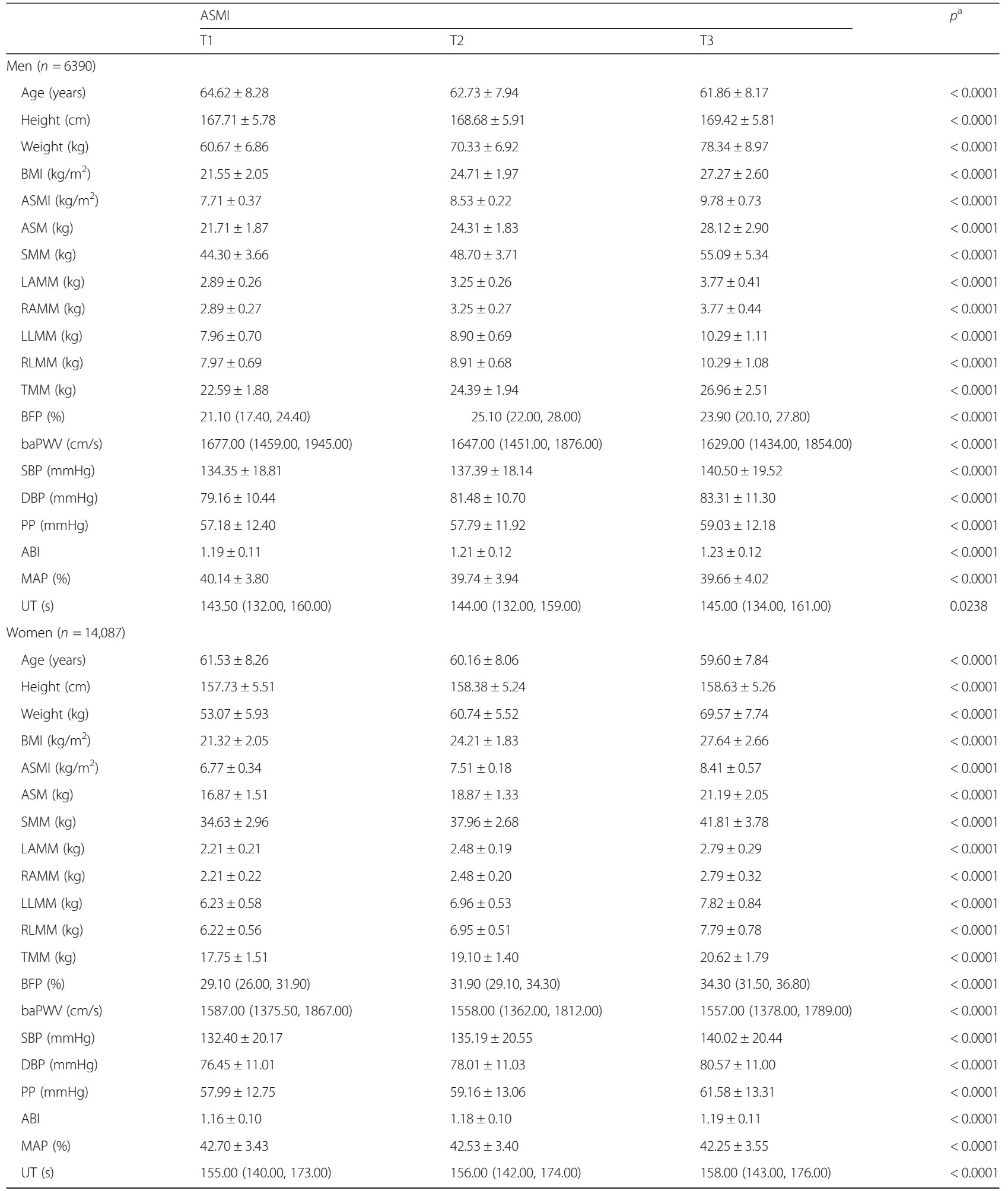

Values are mean \pm SD or medians (25th and 75 th percentile)

Abbreviations: BMI Body mass index, SMM Total muscle mass, LAMM Left arm muscle mass, RAMM Right arm muscle mass, LLMM Left arm muscle mass, RLMM Right leg muscle mass, TMM Trunk muscle mass, BFP total body fat percentage, ASM Appendicular skeletal muscle, ASMI Appendicular skeletal muscle index, baPWV Brachial-ankle pulse wave velocity, SBP systolic blood pressure, DBP diastolic blood pressure, $P P$ Pulse pressure, $A B I$ Ankle brachial index, $M A P$ Mean artery pressure, UT Upstroke time

${ }^{a}$ Test for difference between tertiles was performed using the Kruskal-Wallis test for non-normally distributed continuous variables and ANOVA analysis for normally distributed continuous variables 
Table 3 Correlation between muscle mass indices and baPW

\begin{tabular}{llllll}
\hline & \multicolumn{2}{l}{ Men $(n=6390)$} & & \multicolumn{2}{l}{ Women $(n=14,087)$} \\
\cline { 2 - 3 } & $r$ & $p$ & & $r$ & $p$ \\
\hline ASMI $\left(\mathrm{kg} / \mathrm{m}^{2}\right)$ & -0.080 & $<0.0001$ & & -0.054 & $<0.0001$ \\
ASM $(\mathrm{kg})$ & -0.115 & $<0.0001$ & & -0.112 & $<0.0001$ \\
SMM $(\mathrm{kg})$ & -0.120 & $<0.0001$ & -0.114 & $<0.0001$ \\
LAMM $(\mathrm{kg})$ & -0.106 & $<0.0001$ & -0.116 & $<0.0001$ \\
RAMM $(\mathrm{kg})$ & -0.107 & $<0.0001$ & -0.115 & $<0.0001$ \\
LLMM $(\mathrm{kg})$ & -0.114 & $<0.0001$ & -0.100 & $<0.0001$ \\
RLMM $(\mathrm{kg})$ & -0.116 & $<0.0001$ & -0.111 & $<0.0001$ \\
TMM $(\mathrm{kg})$ & -0.123 & $<0.0001$ & -0.114 & $<0.0001$ \\
\hline
\end{tabular}

Pearson's correlation coefficient was calculated using bivariate correlation analysis

The natural logarithm was applied for baPWV

Abbreviations: baPWV Brachial-ankle pulse wave velocity, ASMI appendicula skeletal muscle index, ASM appendicular skeletal muscle, SMM Total muscle mass, LAMM Left arm muscle mass, RAMM Right arm muscle mass, LLMM Left arm muscle mass, RLMM Right leg muscle mass, TMM Trunk muscle mass

diabetes, negative correlation was observed between cfPWV and fat free mass in left leg in all, right arm, left arm, truncal fat free mass in men and right leg fat free mass in women [19]. In addition, mid-thigh muscle cross-sectional area was found to be an independent determinant of baPWV in middle-aged to elderly Japanese men [16]. Our study firstly found that arm and leg muscle mass on both sides and trunk muscle mass were both negatively associated with baPWV in Chinese community-dwelling men and women aged 45 years and older. Reasons for the controversial results may lie in: 1) the different indicators used for assessing arterial stiffness: cfPWV reflects central arterial stiffness and baPWV reflects both central and peripheral arterial stiffness [36], 2) age-related muscle mass decline (onset of sarcopenia), which is mainly characterized by the loss of appendicular muscle mass [37]. More studies are needed to confirm the associations between muscle mass quantities of different regions and arterial stiffness.

Although previous studies reported liner association between muscle mass indices and baPWV, to our knowledge, this was the first study performed multiple logistic regression analysis to examine the potential effect of muscle mass indices on arterial stiffness (baPWV as the dependent variable). Since arterial stiffness is a predictor for future cardiovascular disease [3], this analysis showed much clinical value. Our study found that the ASMI increased by 0.98 and $0.78 \mathrm{~kg} / \mathrm{m}^{2}$, the risks of developing arterial stiffness decreased by 27.0 and $36.6 \%$ in men and women, respectively. The indices of muscle mass quantity increased by $1 \mathrm{SD}$, the risks of developing arterial stiffness decreased from 5.9\% (SMM) to 52.0\% (LAMM) in men and from $10.0 \%$ (SMM) to $65.2 \%$

Table 4 Multiple linear regression for association between muscle mass indices and baPW adjusted for potential confounders

\begin{tabular}{|c|c|c|c|c|c|c|}
\hline & \multicolumn{3}{|c|}{ Model 1} & \multicolumn{3}{|c|}{ Model 2} \\
\hline & $\overline{R^{2}}$ & $\beta(S E)$ & $p$ & $\mathrm{R}^{2}$ & $\beta(\mathrm{SE})$ & $p$ \\
\hline \multicolumn{7}{|l|}{ Men } \\
\hline ASMI $\left(\mathrm{kg} / \mathrm{m}^{2}\right)$ & 0.172 & $-0.040(0.020)$ & 0.0431 & 0.471 & $-0.208(0.016)$ & $<0.0001$ \\
\hline ASM (kg) & 0.173 & $-0.061(0.016)$ & 0.0002 & 0.473 & $-0.200(0.016)$ & $<0.0001$ \\
\hline SMM (kg) & 0.172 & $-0.053(0.018)$ & 0.0033 & 0.472 & $-0.232(0.020)$ & $<0.0001$ \\
\hline LAMM (kg) & 0.173 & $-0.062(0.015)$ & $<0.0001$ & 0.471 & $-0.176(0.015)$ & $<0.0001$ \\
\hline RAMM (kg) & 0.174 & $-0.062(0.015)$ & $<0.0001$ & 0.471 & $-0.164(0.015)$ & $<0.0001$ \\
\hline LLMM (kg) & 0.173 & $-0.057(0.016)$ & 0.0003 & 0.473 & $-0.198(0.016)$ & $<0.0001$ \\
\hline RLMM (kg) & 0.173 & $-0.058(0.016)$ & 0.0003 & 0.472 & $-0.198(0.017)$ & $<0.0001$ \\
\hline TMM (kg) & 0.172 & $-0.037(0.020)$ & 0.0676 & 0.469 & $-0.255(0.025)$ & $<0.0001$ \\
\hline \multicolumn{7}{|l|}{ Women } \\
\hline ASMI $\left(\mathrm{kg} / \mathrm{m}^{2}\right)$ & 0.288 & $0.001(0.014)$ & 0.9294 & 0.579 & $-0.245(0.012)$ & $<0.0001$ \\
\hline ASM (kg) & 0.288 & $-0.026(0.012)$ & 0.0266 & 0.579 & $-0.206(0.013)$ & $<0.0001$ \\
\hline SMM (kg) & 0.288 & $-0.006(0.013)$ & 0.6521 & 0.578 & $-0.244(0.016)$ & $<0.0001$ \\
\hline LAMM (kg) & 0.288 & $-0.041(0.011)$ & 0.0002 & 0.579 & $-0.178(0.011)$ & $<0.0001$ \\
\hline RAMM (kg) & 0.288 & $-0.040(0.011)$ & 0.0002 & 0.577 & $-0.155(0.011)$ & $<0.0001$ \\
\hline LLMM (kg) & 0.288 & $-0.017(0.011)$ & 0.1317 & 0.578 & $-0.187(0.012)$ & $<0.0001$ \\
\hline RLMM (kg) & 0.288 & $-0.022(0.012)$ & 0.0594 & 0.579 & $-0.203(0.013)$ & $<0.0001$ \\
\hline TMM (kg) & 0.288 & $0.025(0.015)$ & 0.0858 & 0.576 & $-0.259(0.020)$ & $<0.0001$ \\
\hline
\end{tabular}

Multiple linear regression. Model 1: adjusted for age. Model 2: further adjusted for BFP, SBP and DBP for the association between ASMI and baPWV, further adjusted for height, BFP, SBP and DBP for the association between ASM, SMM, LAMM, RAMM, LLMM, RLMM, TMM and baPWV Abbreviations: baPWV Brachial-ankle pulse wave velocity, SMM Total muscle mass, ASMI Appendicular skeletal muscle index, ASM Appendicular skeletal muscle, LAMM Left arm muscle mass, RAMM Right arm muscle mass, LLMM Left arm muscle mass, RLMM Right leg muscle mass, TMM Trunk muscle mass 
Table 5 Multiple logistic regression for the association between muscle mass indices and arterial stiffness (0: baPW $\leq 1800,1$ : baPWV > 1800) adjusted for potential confounders

\begin{tabular}{|c|c|c|c|c|}
\hline & \multicolumn{2}{|l|}{ Model 1} & \multicolumn{2}{|l|}{ Model 2} \\
\hline & OR $(95 \% \mathrm{Cl})$ & $p$ & OR (95\% Cl) & p \\
\hline \multicolumn{5}{|l|}{ Men } \\
\hline ASMI $\left(k g / m^{2}\right)$ & $0.942(0.889,0.997)$ & 0.0391 & $0.731(0.683,0.783)$ & $<0.0001$ \\
\hline ASM (kg) & $0.976(0.960,0.992)$ & 0.0033 & $0.899(0.878,0.921)$ & $<0.0001$ \\
\hline SMM (kg) & $0.989(0.980,0.998)$ & 0.0220 & $0.941(0.927,0.955)$ & $<0.0001$ \\
\hline LAMM (kg) & $0.818(0.727,0.919)$ & 0.0007 & $0.480(0.406,0.568)$ & $<0.0001$ \\
\hline RAMM (kg) & $0.819(0.731,0.919)$ & 0.0006 & $0.510(0.433,0.599)$ & $<0.0001$ \\
\hline LLMM (kg) & $0.941(0.900,0.983)$ & 0.0067 & $0.755(0.709,0.805)$ & $<0.0001$ \\
\hline RLMM (kg) & $0.941(0.900,0.984)$ & 0.0078 & $0.757(0.709,0.808)$ & $<0.0001$ \\
\hline TMM (kg) & $0.985(0.965,1.006)$ & 0.1593 & $0.876(0.844,0.908)$ & $<0.0001$ \\
\hline \multicolumn{5}{|l|}{ Women } \\
\hline ASMI (kg/m²) & $0.949(0.899,1.001)$ & 0.0551 & $0.634(0.594,0.677)$ & $<0.0001$ \\
\hline ASM (kg) & $0.974(0.957,0.992)$ & 0.0037 & $0.844(0.820,0.869)$ & $<0.0001$ \\
\hline SMM (kg) & $0.992(0.982,1.002)$ & 0.1065 & $0.900(0.886,0.914)$ & $<0.0001$ \\
\hline LAMM (kg) & $0.794(0.699,0.902)$ & 0.0004 & $0.348(0.286,0.425)$ & $<0.0001$ \\
\hline RAMM (kg) & $0.807(0.714,0.914)$ & 0.0007 & $0.397(0.329,0.480)$ & $<0.0001$ \\
\hline LLMM (kg) & $0.946(0.903,0.990)$ & 0.0158 & $0.668(0.621,0.719)$ & $<0.0001$ \\
\hline RLMM (kg) & $0.937(0.893,0.982)$ & 0.0068 & $0.616(0.573,0.661)$ & $<0.0001$ \\
\hline TMM (kg) & $1.001(0.979,1.023)$ & 0.9587 & $0.795(0.766,0.826)$ & $<0.0001$ \\
\hline
\end{tabular}

Multiple logistic regression. Model 1: adjusted for age. Model 2: further adjusted for BFP, SBP and DBP for the association between ASMI and baPWV, further adjusted for height, BFP, SBP and DBP for the association between ASM, SMM, LAMM, RAMM, LLMM, RLMM, TMM and baPWV

Abbreviations: baPWV Brachial-ankle pulse wave velocity, SMM Total muscle mass, ASMI Appendicular skeletal muscle index, ASM Appendicular skeletal muscle, LAMM Left arm muscle mass, RAMM Right arm muscle mass, LLMM Left arm muscle mass, RLMM Right leg muscle mass, TMM Trunk muscle mass

(LAMM) in women. The effect of arm muscle mass quantity on risk of arterial stiffness was more pronounced.

It is well known that CVD brings out huge medical and financial burden because of its high mortality [1]. Arterial stiffness, a predictor of future CVD events [3], shows a high prevalence in middle-aged Chinese [30]. Meanwhile, the quantity of total skeletal muscle mass of Chinese adults was at a low level [31]. The results above suggested that participants with higher muscle mass quantity have low risk for developing arterial stiffness, which indicated that taking actions to preserve muscle mass might have potential benefits for prevention of cardiovascular disease. More specific, the findings indicated that: 1) not only men but also women should take actions to preserve muscle mass; 2) muscle loss should be monitored at an earlier age of 45 rather than the elderly; 3) medical staff in community should pay more attention on arm muscle mass in solutions for maintaining muscle quantity such as resistance training. The possible influences of our findings on programmatic interventions of public health are as follows: Among Chinese community residents aged 45 years and older, 1) quantity of muscle mass should be regularly screened, since loss of muscle mass is difficult to be perceived without obvious decline of muscle function, 2) more clinical or community programs for improving level of muscle mass should be implemented such as some courses of nutrition and physical training.

Several mechanisms may involve in the association between muscle mass and arterial stiffness. Firstly, loss of muscle mass was considered as a cause of insulin resistance by impairing glucose metabolism [21]. Evidence suggested that insulin resistance could determine the arterial elastic properties and coronary flow reserve [22] and played an important role in the development of atherosclerotic cardiovascular disease [38]. Secondly, chronic inflammation was widely studied as a pathway both to muscle loss and arterial stiffness. Results from a cohort study in Chinese sarcopenia patients showed that high levels of the inflammatory cytokines TWEAK and TNF- $\alpha$ were associated with an increased risk of sarcopenia, while the insulin growth factor 1 , insulin, and adiponectin were associated with a decreased risk of sarcopenia [23]. Meanwhile, these mediators of inflammation had been examined as biomarkers and causal agents in the pathogenesis of arterial stiffness [24] and atherosclerosis, and they had been considered as a therapeutic target for cardiovascular disease [39]. These studies supported that loss of muscle mass might be a risk 
factor of arterial stiffness and further affected the development of CVD. However, experimental and clinical studies are warranted.

There are some limitations in the present study. First, due to the cross-sectional design of our study, we cannot make inferences about causality which warrants further prospective and interventional researches. Secondly, bioelectrical impedance analysis (BIA) but not Dual energy $\mathrm{X}$-ray absorptiometry (DEXA, considered as gold standard) was used to measure body composition. However, evidence have shown that body composition measured by BIA were strongly correlated with that measured by DEXA [40] and have been widely used in epidemiological and clinical studies [31, 41]. In addition, compared with DEXA, BIA can avoid the risk of exposure to ionized radiation and be more practical and economical for studies with a large sample. Finally, the impact of blood biomarkers were not evaluated which evidences have shown to be associated with arterial stiffness [42-44].

\section{Conclusion}

In conclusion, our study indicated that low muscle mass was associated with increased risk of arterial stiffness in Chinese community-dwelling adults aged 45 years and older. Further longitudinal researches are needed to confirm the potential effects of muscle mass on arterial stiffness observed in this study for the prevention of cardiovascular disease.

\footnotetext{
Abbreviations

ABI: Ankle brachial index; ASM: Appendicular skeletal muscle mass; ASMI: Appendicular skeletal muscle mass index; baPW: Brachial-ankle pulse wave velocity; BFP: Percentage of body fat; BIA: Bioelectrical impedance analysis; BMI: Body mass index; CfPWV: Carotid-femoral pulse wave velocity; CVD: Cardiovascular disease; DBP: Diastolic blood pressure; DEXA: Dual energy X-ray absorptiometry; LAMM: Muscle mass of the left arm; LLMM: Muscle mass of left leg; MAP: Mean artery pressure; PP: Pulse pressure; PWV: Pulse wave velocity; RAMM: Muscle mass of right arm; RLMM: Muscle mass of right leg; SBP: Systolic blood pressure; SD: Standard deviation; SMM: Total skeletal muscle mass; TMM: Muscle mass of trunk; UT: Upstroke time
}

\section{Acknowledgements}

The authors thank the subjects who had participated in this study.

\section{Authors' contributions}

MY, JC and XZ contributed to the study conception and design. Material preparation, data collection and analysis were performed by ZD, MY and FW. The first draft of the manuscript was written by MY and XZ, JC, YW and CJ commented and modified on previous versions of the manuscript. All authors read and approved the final manuscript.

\section{Funding}

This study was funded by the Nutrition Scientific Research Foundation of BYHEALTH [No. TY0171112] and the National Natural Science Foundation of China [Grant No. 31601472]. The Nutrition Scientific Research Foundation of BY-HEALTH [No. TY0171112] is used to support design of the study and data collection, and the National Natural Science Foundation of China [Grant No. 31601472 is used to support data analysis and interpretation in writing the manuscript.

\section{Availability of data and materials}

The datasets generated and/or analyzed during the current study are not publicly available due the government policy for original Chinese biodata of Chinese population but are available from the corresponding author on reasonable request.

\section{Ethics approval and consent to participate}

The study was approved by Ethics Committee of Chinese Clinical Trial Registry. All participants gave written informed consent.

\section{Consent for publication}

Not applicable.

\section{Competing interests}

The authors declare that they have no competing interests.

Received: 25 August 2019 Accepted: 5 February 2020

Published online: 13 February 2020

\section{References}

1. Liu S, Li Y, Zeng X, Wang H, Yin P, Wang L, Liu Y, Liu J, Qi J, Ran S et al. Burden of Cardiovascular Diseases in China, 1990-2016: Findings From the 2016 Global Burden of Disease Study. JAMA Cardiol. 2019;4(4):342-52.

2. Palombo C, Kozakova M. Arterial stiffness, atherosclerosis and cardiovascular risk: pathophysiologic mechanisms and emerging clinical indications. Vasc Pharmacol. 2016;77:1-7.

3. Vlachopoulos C, Aznaouridis K, Stefanadis C. Prediction of cardiovascular events and all-cause mortality with arterial stiffness: a systematic review and meta-analysis. J Am Coll Cardiol. 2010;55(13):1318-27.

4. Ohkuma T, Ninomiya T, Tomiyama H, Kario K, Hoshide $S$, Kita $Y$, Inoguchi T, Maeda Y, Kohara K, Tabara Y, et al. Brachial-Ankle Pulse Wave Velocity and the Risk Prediction of Cardiovascular Disease: An Individual Participant Data Meta-Analysis. Hypertension. 2017;69(6):1045-52.

5. Takashima N, Turin TC, Matsui K, Rumana N, Nakamura $Y$, Kadota A, Saito $Y$, Sugihara H, Morita Y, Ichikawa M, et al. The relationship of brachial-ankle pulse wave velocity to future cardiovascular disease events in the general Japanese population: the Takashima study. J Hum Hypertens. 2014;28(5): 323-7.

6. Katakami N, Osonoi T, Takahara M, Saitou M, Matsuoka TA, Yamasaki Y, Shimomura I. Clinical utility of brachial-ankle pulse wave velocity in the prediction of cardiovascular events in diabetic patients. Cardiovasc Diabetol. 2014;13:128

7. Tomiyama H, Matsumoto C, Shiina K, Yamashina A. Brachial-ankle PWV: current status and future directions as a useful marker in the Management of Cardiovascular Disease and/or cardiovascular risk factors. J Atheroscler Thromb. 2016;23(2):128-46.

8. Ikonomidis I, Makavos G, Lekakis J. Arterial stiffness and coronary artery disease. Curr Opin Cardiol. 2015;30(4):422-31.

9. Ferreira I, Snijder MB, Twisk JW, van Mechelen W, Kemper HC, Seidell JC, Stehouwer CD. Central fat mass versus peripheral fat and lean mass: opposite (adverse versus favorable) associations with arterial stiffness? The Amsterdam growth and health longitudinal study. J Clin Endocrinol Metab. 2004;89(6):2632-9.

10. Lin LY, Liao YC, Lin HF, Lee YS, Lin RT, Hsu CY, Juo SH. Determinants of arterial stiffness progression in a Han-Chinese population in Taiwan: a 4-year longitudinal follow-up. BMC Cardiovasc Disord. 2015;15:100.

11. Wohlfahrt P, Somers VK, Sochor O, Kullo I, Jean N, Lopez-Jimenez F. Influence of body fatness distribution and total lean mass on aortic stiffness in nonobese individuals. Am J Hypertens. 2015;28(3):401-8.

12. Diemer FS, Brewster LM, Haan YC, Oehlers GP, van Montfrans GA, Nahar-van Venrooij LMW. Body composition measures and cardiovascular risk in highrisk ethnic groups. Clin Nutr. 2019;38(1):450-6.

13. Fu S, Luo L, Ye P, Liu Y, Zhu B, Zheng J, Bai Y, Bai J. Overall and abdominal obesity indicators had different association with central arterial stiffness and hemodynamics independent of age, sex, blood pressure, glucose, and lipids in Chinese community-dwelling adults. Clin Interv Aging. 2013:8:1579-84.

14. Snijder MB, Henry RM, Visser M, Dekker JM, Seidell JC, Ferreira I, Bouter LM, Yudkin JS, Westerhof N, Stehouwer CD. Regional body composition as a determinant of arterial stiffness in the elderly: the Hoorn study. J Hypertens. 2004;22(12):2339-47. 
15. Zhang $L$, Guo Q, Feng BL, Wang $C Y$, Han PP, Hu J, Sun XD, Zeng WF, Zheng ZX, $\mathrm{Li} \mathrm{HS}$, et al. A cross-sectional study of the association between arterial stiffness and sarcopenia in Chinese community-dwelling elderly using the Asian working group for sarcopenia criteria. J Nutr Health Aging. 2019;23(2):195-201.

16. Ochi M, Kohara K, Tabara Y, Kido T, Uetani E, Ochi N, Igase M, Miki T. Arterial stiffness is associated with low thigh muscle mass in middle-aged to elderly men. Atherosclerosis. 2010;212(1):327-32.

17. Sanada K, Miyachi M, Tanimoto M, Yamamoto K, Murakami H, Okumura S, Gando Y, Suzuki K, Tabata I, Higuchi M. A cross-sectional study of sarcopenia in Japanese men and women: reference values and association with cardiovascular risk factors. Eur J Appl Physiol. 2010;110(1):57-65.

18. Abbatecola AM, Chiodini P, Gallo C, Lakatta E, Sutton-Tyrrell K, Tylavsky FA, Goodpaster B, de Rekeneire N, Schwartz AV, Paolisso G, et al. Pulse wave velocity is associated with muscle mass decline: Health $A B C$ study. Age (Dordr). 2012;34(2):469-78.

19. Anoop S, Misra A, Bhardwaj S, Gulati S. High body fat and low muscle mass are associated with increased arterial stiffness in Asian Indians in North India. J Diabetes Complicat. 2015;29(1):38-43.

20. Liu X, Zheng X, Yi C, Wu J, Ye H, Guo Q, Yu X, Yang X. Gender-specific associations of skeletal muscle mass and arterial stiffness among peritoneal dialysis patients. Sci Rep. 2018;8(1):1351.

21. Aleman-Mateo H, Lopez Teros MT, Ramirez FA, Astiazaran-Garcia H. Association between insulin resistance and low relative appendicular skeletal muscle mass: evidence from a cohort study in community-dwelling older men and women participants. J Gerontol A Biol Sci Med Sci. 2014; 69(7):871-7.

22. Ikonomidis I, Lambadiari V, Pavlidis G, Koukoulis C, Kousathana F, Varoudi M, Spanoudi F, Maratou E, Parissis J, Triantafyllidi $H$, et al. Insulin resistance and acute glucose changes determine arterial elastic properties and coronary flow reserve in dysglycaemic and first-degree relatives of diabetic patients. Atherosclerosis. 2015;241(2):455-62.

23. Li CW, Yu K, Shyh-Chang N, Li GX, Jiang LJ, Yu SL, Xu LY, Liu RJ, Guo ZJ, Xie $H Y$, et al. Circulating factors associated with sarcopenia during ageing and after intensive lifestyle intervention. J Cachexia Sarcopenia Muscle. 2019; 10(3):586-600.

24. Kim M, Kim M, Yoo HJ, Lee SY, Lee SH, Lee JH. Age-specific determinants of pulse wave velocity among metabolic syndrome components, inflammatory markers, and oxidative stress. J Atheroscler Thromb. 2018;25(2):178-85.

25. Gerhard-Herman MD, Gornik HL, Barrett C, Barshes NR, Corriere MA, Drachman DE, Fleisher LA, Fowkes FG, Hamburg NM, Kinlay S, et al. 2016 AHA/ACC guideline on the Management of Patients with Lower Extremity Peripheral Artery Disease: executive summary: a report of the American College of Cardiology/American Heart Association task force on clinical practice guidelines. Circulation. 2017;135(12):e686-725.

26. Dmitruk A, Czeczelewski J, Czeczelewska E, Golach J, Parnicka U. Body composition and fatty tissue distribution in women with various menstrual status. Rocz Panstw Zakl Hig. 2018;69(1):95-101.

27. Sterkowicz S, Jaworski J, Lech G, Palka T, Sterkowicz-Przybycien K, Bujas P, Pieta P, Moscinski Z. Effect of acute effort on isometric strength and body balance: trained vs. Untrained Paradigm. PLoS One. 2016;11(5):e0155985.

28. Yamashina A, Tomiyama H, Takeda K, Tsuda H, Arai T, Hirose K, Koji Y, Hori $S$, Yamamoto $Y$. Validity, reproducibility, and clinical significance of noninvasive brachial-ankle pulse wave velocity measurement. Hypertens Res. 2002;25(3):359-64

29. Munakata M. Brachial-Ankle Pulse Wave Velocity: Background, Method, and Clinical Evidence. Pulse (Basel). 2016;3(3-4):195-204.

30. Wang JW, Zhou ZQ, Hu DY. Prevalence of arterial stiffness in North China, and associations with risk factors of cardiovascular disease: a communitybased study. BMC Cardiovasc Disord. 2012;12:119.

31. Wu M, Wei YX, Yu CQ, Du HD, Lyu J, Guo Y, Bian Z, Tan YL, Pei P, Chen JS, et al. levels of skeletal muscle mass and handgrip strength in adults from 10 regions of China. Zhonghua liu xing bing xue za zhi $=$ Zhonghua liuxingbingxue zazhi. 2019;40(4):376-81.

32. Corona G, Giagulli VA, Maseroli E, Vignozzi L, Aversa A, Zitzmann M, Saad F, Mannucci E, Maggi M. Testosterone supplementation and body composition: results from a meta-analysis of observational studies. J Endocrinol Investig. 2016;39(9):967-81.

33. Hougaku H, Fleg JL, Najjar SS, Lakatta EG, Harman SM, Blackman MR, Metter EJ. Relationship between androgenic hormones and arterial stiffness, based on longitudinal hormone measurements. Am J Phys Endocrinol Metab. 2006;290(2):E234-42
34. Schorr M, Dichtel LE, Gerweck AV, Valera RD, Torriani M, Miller KK, Bredella MA. Sex differences in body composition and association with cardiometabolic risk. Biol Sex Differ. 2018;9(1):28.

35. Bredella MA. Sex differences in body composition. Adv Exp Med Biol. 2017; 1043:9-27.

36. Luo KQ, Feng XW, Xu BC, Long HB. Association between arterial stiffness and risk of coronary artery disease. Pak J Med Sci. 2014;30(6):1314-8.

37. Cruz-Jentoft AJ, Baeyens JP, Bauer JM, Boirie Y, Cederholm T, Landi F, Martin FC, Michel JP, Rolland Y, Schneider SM, et al. Sarcopenia: European consensus on definition and diagnosis: report of the European working group on sarcopenia in older people. Age Ageing. 2010;39(4):412-23.

38. Di Pino A, DeFronzo RA. Insulin resistance and atherosclerosis: implications for insulin sensitizing agents. Endocr Rev. 2019;40(6):1447-67.

39. Golia E, Limongelli G, Natale F, Fimiani F, Maddaloni V, Pariggiano I, Bianchi R, Crisci M, D'Acierno L, Giordano R, et al. Inflammation and cardiovascular disease: from pathogenesis to therapeutic target. Curr Atheroscler Rep. 2014;16(9):435.

40. McLester CN, Nickerson BS, Kliszczewicz BM, McLester JR. Reliability and agreement of various InBody body composition analyzers as compared to dual-energy $\mathrm{X}$-ray absorptiometry in healthy men and women. J Clin Densitom. 2018;S1094-6950(18):30221-X.

41. Xiao J, Purcell SA, Prado CM, Gonzalez MC. Fat mass to fat-free mass ratio reference values from NHANES III using bioelectrical impedance analysis. Clin Nutr. 2018;37(6 Pt A):2284-7.

42. Liu X, Sun N, Yu T, Fan F, Zheng M, Qian G, Wang B, Wang Y, Tang G, Li J, et al. The independent and joint Association of Blood Pressure, serum Total Homocysteine, and fasting serum glucose levels with brachial-ankle pulse wave velocity in Chinese hypertensive adults. Int Heart J. 2016;57(5):627-33.

43. Zhan B, Huang X, Wang J, Qin X, Zhang J, Cao J, Song Y, Liu L, Li P, Yang R, et al. Association between lipid profiles and arterial stiffness in Chinese patients with hypertension: insights from the CSPPT. Angiology. 2019;70(6): 515-22.

44. Zhao W, Gong W, Wu N, Li Y, Ye K, Lu B, Zhang Z, Qu S, Li Y, Yang Y, et al, Association of lipid profiles and the ratios with arterial stiffness in middleaged and elderly Chinese. Lipids Health Dis. 2014;13:37.

\section{Publisher's Note}

Springer Nature remains neutral with regard to jurisdictional claims in published maps and institutional affiliations.

Ready to submit your research? Choose BMC and benefit from

- fast, convenient online submission

- thorough peer review by experienced researchers in your field

- rapid publication on acceptance

- support for research data, including large and complex data types

- gold Open Access which fosters wider collaboration and increased citations

- maximum visibility for your research: over $100 \mathrm{M}$ website views per year

At $\mathrm{BMC}$, research is always in progress.

Learn more biomedcentral.com/submissions 